\title{
SUPERCONDUCTING PLATE IN TRANSVERSE MAGNETIC FIELD: NEW STATE
}

\author{
E.G. Batyev* \\ A.V. Rzhanov Institute of Semiconductor Physics SB RAS, \\ 630090, Novosibirsk, Russia.
}

November 15, 2018

\begin{abstract}
A model to describe Cooper pairs near the transition point (on temperature and magnetic field), when the distance between them is big compared to their sizes, is proposed. A superconducting plate whose thickness is less than the pair size in the transverse magnetic field near the critical value $H_{c 2}$ is considered as an application of the model. A new state that is energetically more favourable than that of Abrikosov vortex state within an interval near the transition point was obtained. The system's wave function in this state looks like that of Laughlin's having been used in fractional quantum Hall effect (naturally, in our case - for Cooper pairs as Bose-particles) and it corresponds to homogeneous incompressible liquid. The state energy is proportional to the first power of value $\left(1-H / H_{c 2}\right)$, unlike the vortex state energy having this value squared. The interval of the new state existence is greater for dirty specimens.
\end{abstract}

PACS 71.27.+a

\section{Introduction}

The difference of free energies of superconducting $F_{S}$ and normal $F_{N}$ states in GinzburgLandau theory is written as:

$$
\begin{array}{r}
F_{S}-F_{N} \rightarrow \int d \mathbf{r}\left\{\frac{1}{2 M}\left|\left(\nabla-i \frac{2 e}{c} \mathbf{A}\right) \Psi(\mathbf{r})\right|^{2}+\right. \\
\left.\alpha|\Psi|^{2}+\frac{\beta}{2}|\Psi|^{4}\right\} .
\end{array}
$$

Only the part explicitly dependent on the order parameter $\Psi(\mathbf{r})$ is considered here.

Value $\Psi(\mathbf{r})$ can be treated as the wave function of Cooper pair (precise to the coefficient). All pairs are in the same state (they form Bose-condensate) and, therefore, a function from

*e-mail:batyev@isp.nsc.ru 
one coordinate is sufficient. Such an interpretation enables us to go further and describe the system of Cooper pairs with the help of Hamiltonian:

$$
\begin{aligned}
\mathbf{H}= & \int d \mathbf{r}\left\{\frac{-1}{2 M} \Psi^{+}(\mathbf{r})\left(\nabla-i \frac{2 e}{c} \mathbf{A}(\mathbf{r})\right)^{2} \Psi(\mathbf{r})+\right. \\
& \left.\alpha \Psi^{+}(\mathbf{r}) \Psi(\mathbf{r})+\frac{\beta}{2} \Psi^{+}(\mathbf{r}) \Psi^{+}(\mathbf{r}) \Psi(\mathbf{r}) \Psi(\mathbf{r})\right\} .
\end{aligned}
$$

Here $\Psi(\mathbf{r}), \Psi^{+}(\mathbf{r})$ are Bose-type operators in the secondary quantization. If we replace them by simply functions (to describe Bose-condensate), then there will be expression (1).

Such a generalization of GL theory was suggested many years ago in contribution [1] but with no success. In the present study it is shown that, in some cases, this approach leads to a new state which is energetically more favourable than that following from a usual formulation of GL theory. Namely, a new state turns up in a quasi-2D case (superconducting plate) in the transverse magnetic field near the upper critical field $H_{c 2}$. Seemingly, it can be proved experimentally.

First on the speculations in favor of a new approach with the use of operator (2). 1) In the work [2], a phase transition in superconductor was considered. The main thing shown in this study is that, near the transition point, the diagram technique for a singular part of two-particle Green function is the same as for Bose-particles system. This singular part just describes the Cooper pair. 2) It is possible to show that precisely the same ratios for coefficients of GL theory, earlier obtained by Gor'kov (see e.g. [3]), follow from the diagram approach used in [2] for the two-particle Green function. That is, first, we compare BoseHamiltonian - for which there appears the same diagram technique as for the two-particle Green function - to Cooper pairs, and then we get the result for Bose-condensate as in GL theory. 3) Finally, the interaction (contribution of fourth order) appears in GL theory due to the thing that Cooper pairs overlap, i.e. because of Pauli principle. If it does not happen, i.e. they are separated, then there is no interaction. It is not seen in the traditional approach because self-action always remains.

Note that the size of a pair does not change with temperature on the order of value, whereas their number decreases. Therefore, one can consider the separated pairs near the transition point.

The presented arguments are a ground for the suggested generalization. It is worth emphasizing that the description of superconducting state as a set of particles (Cooper pairs) - as it is proposed - appears to be natural, but it is not strictly grounded and, thus, it is no more than a model.

\section{Discussion of the model}

Let a superconducting plate be in the transverse magnetic field $\mathrm{H}$. Abrikosov vortex lattice forms near the field $H_{c 2}$. The lattice can melt analogously to any crystal melting. When discussing melting (see e.g. Review [3]), vortices are thought to remain, but the long range order of their arrangement disappears. It turns out that a liquid state of other type is possible near $H_{c 2}$ and it is different from all considered earlier [3]. Namely, there are no vortices but there is a homogeneous system - liquid of Cooper pairs (without Bose-condensate and vorticies). Such a picture naturally appears in Cooper pairs description assisted with the Hamiltonian (2). 
First it is necessary to determine what to consider as particles (Cooper pairs) concentration. It would be necessary to think $|\Psi|^{2}$ as such one, i.e. without external fields $|\alpha| / \beta$ (in the unit of volume of a 3D case). However, in GL theory, this value is not determined because any value, different from $\Psi$ in its constant multiplier, may be taken as an order parameter, lest the physical values should not be unchanged. These values are: 1) magnetic field penetration depth

$$
\lambda(T)=\sqrt{\frac{M c^{2} \beta}{4 \pi(2 e)^{2}|\alpha|}},
$$

2) length of coherence

$$
\xi(T)=\frac{1}{\sqrt{2 M|\alpha|}},
$$

3) free energy change under the transition into ordered state

$$
\frac{\delta F}{V}=-\frac{\alpha^{2}}{2 \beta} \rightarrow-\frac{A^{2} \gamma T_{c}^{2}}{2}\left(1-T / T_{c}\right)^{2}
$$

(the presented value is from the microscopic theory, $\gamma$ is density of states on Fermi surface, $A \approx 3.06$ ). It is seen from these expressions that only two were fixed (all is clear with the charge), out of these three GL theory parameters $\alpha, \beta, M$. As for the rest, there is arbitrariness and ratio $|\alpha| / \beta$ remains indefinite, which is irrelevant for the GL approach. It is common knowledge.

In the operator formulation (2), when we say about particles (Cooper pairs) and their quantity, it is necessary to register the left parameter $|\alpha| / \beta$ which just corresponds to particles density. For this purpose it is necessary to estimate the number of Cooper pairs $N_{C P}$. Let us show the way it is possible to make it.

Consider a usual superconductor. Here it is clear how to estimate it. First, let us present some known data from the theory of superconductivity which are necessary for estimations. In Bardeen-Cooper-Schrieffer model (only electrons attraction with opposite momenta and spins are considered in the Hamiltonian), the transition from electron operators $a_{\mathbf{p} \sigma}$ to quasi-particles operators $\alpha_{\mathbf{p} \sigma}$ is realized by Bogolyubov transformations:

$$
\begin{gathered}
a_{\mathbf{p} \uparrow}=u_{\mathbf{p}} \alpha_{\mathbf{p} \uparrow}+v_{\mathbf{p}} \alpha_{-\mathbf{p} \downarrow}^{+}, \quad a_{-\mathbf{p} \downarrow}=u_{\mathbf{p}} \alpha_{-\mathbf{p} \downarrow}-v_{\mathbf{p}} \alpha_{\mathbf{p} \uparrow}^{+}, \\
\left(u_{\mathbf{p}}^{2}, v_{\mathbf{p}}^{2}\right)=\frac{1}{2}\left(1 \pm \frac{\xi_{\mathbf{p}}}{\epsilon_{\mathbf{p}}}\right), \quad u_{\mathbf{p}} v_{\mathbf{p}}=\frac{-\Delta}{2 \epsilon_{\mathbf{p}}},
\end{gathered}
$$

where $\epsilon_{\mathbf{p}}=\sqrt{\xi_{\mathbf{p}}^{2}+\Delta^{2}}$ is quasi-particles spectrum, $\xi_{\mathbf{p}}$ is electron energy calculated from Fermi energy.

Consider the mean value $<\Psi_{\uparrow}(\mathbf{r}) \Psi_{\downarrow}\left(\mathbf{r}^{\prime}\right)>$. It is proportional to the electron wave function in a pair. If we calculate the double-integral from the square of this value's module, then there will be the number of electrons in pairs (with the spin upwards and down), i.e. pairs doubled number. Thus,

$$
N_{C P}(T)=\frac{1}{2} \int d \mathbf{r} d \mathbf{r}^{\prime}\left|<\Psi_{\uparrow}(\mathbf{r}) \Psi_{\downarrow}\left(\mathbf{r}^{\prime}\right)>\right|^{2} .
$$

Here, operators $\Psi_{\uparrow, \downarrow}$ are usual field electron operators, e.g.:

$$
\Psi_{\uparrow}(\mathbf{r})=\frac{1}{\sqrt{V}} \sum_{\mathbf{p}} a_{\mathbf{p} \uparrow} \exp (i \mathbf{p r}) .
$$


Using the transition to quasi-particles operators and calculating the mean value, we have:

$$
\begin{aligned}
&<\Psi_{\uparrow}(\mathbf{r}) \Psi_{\downarrow}\left(\mathbf{r}^{\prime}\right)>=\frac{1}{V} \sum_{\mathbf{p}} \frac{\Delta}{2 \epsilon_{\mathbf{p}}} \tanh \left\{\frac{\epsilon_{\mathbf{p}}}{2 T}\right\} \times \\
& \times \exp \left[i \mathbf{p}\left(\mathbf{r}-\mathbf{r}^{\prime}\right)\right] .
\end{aligned}
$$

Integration on co-ordinates in value $N_{C P}(T)$ gives multiplier $V^{2} \delta_{\mathbf{p}, \mathbf{q}}(\mathbf{p}, \mathbf{q}$ are momenta in different sums); so, finally we will get:

$$
N_{C P}(T)=\frac{1}{2} \sum_{\mathbf{p}} \frac{\Delta^{2}}{4 \epsilon_{\mathbf{p}}^{2}} \tanh ^{2}\left\{\frac{\epsilon_{\mathbf{p}}}{2 T}\right\} .
$$

At zero temperature we have:

$$
N_{C P}(0) / V=\frac{\gamma \Delta_{0}}{2} \frac{\pi}{4}
$$

Near the transition point there is:

$$
\begin{array}{r}
\frac{|\alpha|}{\beta} \equiv N_{C P}\left(T \approx T_{c}\right) / V=\frac{\gamma \Delta^{2}}{8 T_{c}} D \\
D \equiv \int_{0}^{\infty} \frac{d x}{x^{2}} \tanh ^{2}(x) \approx 1.7 .
\end{array}
$$

As is known the gap in the spectrum near transition point is

$$
\Delta=A T_{c} \sqrt{1-T / T_{c}} .
$$

This value, just as ratio $\alpha^{2} / \beta$ (see (5)), does not change in dirty superconductor (Anderson theorem). Besides, the expression for pairs number (9) remains the same. It turns out that, with impurities, only mass $M$ changes (see below (12)).

It is not difficult to get mass $M_{0}$ (for a pure case) assisted with expressions for other constants. For instance, for $\lambda(T)$ (see $(3))$ we have:

$$
\lambda(T)=\frac{\lambda(0)}{\sqrt{2\left(1-T / T_{c}\right)}}, \quad \lambda(0)=\sqrt{\frac{m c^{2}}{4 \pi n e^{2}}},
$$

where the known ratio of superfluid component near the transition point for pure superconductor ( $m$ - electron mass, $n$ - concentration) is used. It is sufficient for our purposes. As a result:

$$
\frac{M_{0}}{m}=\frac{3 A^{2} D}{16} \frac{T_{c}}{\epsilon_{F}}, \quad \frac{M}{M_{0}}=\frac{\kappa}{\kappa_{0}} \sim \frac{\xi_{0}}{l}\left(l<\xi_{0}\right) .
$$

Here $\kappa=\lambda(T) / \xi(T)$ is a GL parameter $\left(k_{0}\right.$ - for pure sample), $l$ - free path length. Let us point out the thing that it is when Cooper pairs number is meant by particles number.

Now one more expression for coherence length (see (31), (44), (5) и (11)):

$$
\xi(T)=\frac{1}{A \sqrt{6}} \frac{v_{F} / T_{c}}{\sqrt{1-T / T_{c}}} .
$$

The last three expressions are for pure superconductor (not considering the expression for $M)$. 
Finally, let us put down the expression for coefficient $\beta$ which is derived from comparison (5) and (9) using (10):

$$
\beta=\left(\frac{8}{A}\right)^{2} \frac{1}{\gamma D^{2}}
$$

Using expression (9), it is possible to get the evaluation of temperature interval near the transition point $\delta T / T_{c}$ when the mean distance between Cooper pairs becomes larger than the size of Cooper pair $\xi_{0}$. For pure superconductor $\xi_{0} \sim v_{F} / T_{c}$, so it makes:

$$
\frac{\delta T}{T_{c}} \sim\left(\frac{T_{c}}{\epsilon_{F}}\right)^{2}
$$

It is in this region that one can consider Cooper pairs as particles; though this interval is still beyond the limits of fluctuation region which occurs near the transition point in the interval $\delta T / T_{c} \sim\left(T_{c} / \epsilon_{F}\right)^{4}$ but it is hardly possible to see anything new here.

\section{New state}

The superconducting plate in transverse magnetic field is analyzed. Let the thickness of plate $d$ is small enough - less than the pair size but more than the distance among electrons:

$$
1 / p_{F}<<d<\xi_{0}, \sqrt{\xi_{0} l}
$$

The last value is the pair size in a dirty sample ( $l$ - free path length). In this case the above-mentioned estimates for a massive sample are suitable but, instead of the Hamiltonian (2), it is necessary to write down a two-dimensional equivalent. Namely:

$$
\begin{aligned}
\mathbf{H} \rightarrow & \int d \mathbf{r}\left\{\frac{-1}{2 M} \Psi^{+}(\mathbf{r})\left(\nabla-i \frac{2 e}{c} \mathbf{A}(\mathbf{r})\right)^{2} \Psi(\mathbf{r})+\right. \\
& \left.\alpha \Psi^{+}(\mathbf{r}) \Psi(\mathbf{r})+\frac{\beta_{2}}{2} \Psi^{+}(\mathbf{r}) \Psi^{+}(\mathbf{r}) \Psi(\mathbf{r}) \Psi(\mathbf{r})\right\} .
\end{aligned}
$$

Two-dimensional integration (in the plate's plane) is implied here and $\beta_{2} \equiv \beta / d$, and all the operators are two-dimensional.

We are focused on the basic state of particle (Cooper pair) in the magnetic field. As is known, in the cylindrical calibration of vector potential $\left(A_{\varphi}=H \rho / 2, A_{\rho}=A_{z}=0\right)$ wave functions of the basic Landau level are registered as:

$$
\phi_{n} \sim z^{n} \exp \left(-\frac{|z|^{2}}{4 a_{H}^{2}}\right), \quad z=x+i y .
$$

Here $n$ has non-negative integral values $(\mathrm{n}=0,1,2 \ldots), a_{H}$ - magnetic length (for Cooper pair $\left.a_{H}^{2}=c /(2|e| H)\right)$. Energy

$$
E_{1}=\frac{1}{2} \frac{2|e| H}{M c}, \quad \frac{|e| H_{c 2}}{M c}=|\alpha|
$$

corresponds to these states.

It is possible to employ a Laughlin-like function [5] (functions of this type were used in the theory of fractional quantum Hall effect) - naturally, regarding Bose-particles. The 
advantage of such functions consists in the thing that short-range interaction is completely excluded, just as in the case we are focused on. Laughlin-like function $\Phi_{L}$, in our case, is the following:

$$
\Phi_{L} \sim \prod_{i>j=1}^{N}\left(z_{i}-z_{j}\right)^{2} \prod_{k=1}^{N} \exp \left(-\frac{\left|z_{k}\right|^{2}}{4 a_{H}^{2}}\right)
$$

( $N$ - number of Cooper pairs). This function is symmetrical in its particles exchange, just as it is required, and the interaction turns to zero. Note that it is possible to analyze any even power instead of square, particles concentration being lower. For function (18) the concentration is maximally possible (one particle per two magnetic flux quanta), i.e. the energy is minimally lower near the transition point $\left(H<H_{c 2}\right)$. This is the state with unconsidered fluctuations (without other Landau levels). As a result, the mean value of operator (16) on state (18) is equal to:

$$
\begin{aligned}
\frac{\langle\mathrm{H}>}{S} & =-|\alpha|\left(1-\frac{H}{H_{c 2}}\right) \frac{|e| H}{2 \pi c} \approx \\
& \approx-|\alpha|\left(1-\frac{H}{H_{c 2}}\right) \frac{|e| H_{c 2}}{2 \pi c},
\end{aligned}
$$

where $S$ is area. It is taken into account that the interaction in this state is equal to zero. For Abrikosov vortex state we have:

$$
\frac{F_{S}-F_{N}}{S}=\frac{-\alpha^{2}}{2 \beta_{2} C}\left(1-\frac{H}{H_{c 2}}\right)^{2} .
$$

Here constant $C$ depends on lattice type (for triangular lattice $C \approx 1.16$ ).

Thus, one should compare two energies:

$$
\frac{-\alpha^{2}}{2 \beta_{2} C}\left(1-\frac{H}{H_{c 2}}\right)^{2} \div-|\alpha|\left(1-\frac{H}{H_{c 2}}\right) \frac{|e| H_{c 2}}{2 \pi c} .
$$

This or that state is realized depending on what energy is lower. Whence forth, there is the magnetic field interval when the state occurs according to Laughlin:

$$
\frac{\delta H}{H_{c 2}}=\frac{M \beta}{d} \frac{C}{\pi} .
$$

Using the previous result we have:

$$
\left(\frac{\delta H}{H_{c 2}}\right)_{0}=\frac{T_{c}}{\epsilon_{F}} \frac{1}{p_{F} d} \frac{24 \pi C}{D} \approx \frac{T_{c}}{\epsilon_{F}} \frac{51.448}{p_{F} d} .
$$

Index 0 means that this expression is for a pure sample (mirror walls of a plate and no impurities). It is a small value for a plate from usual superconductors even with a large numerical coefficient.

In case of a dirty sample (free path length is small $l<<\xi_{0}, d$ ), there occurs an additional multiplier:

$$
\frac{\delta H}{H_{c 2}}=\left(\frac{\delta H}{H_{c 2}}\right)_{0} \frac{\kappa}{\kappa_{0}} \sim\left(\frac{\delta H}{H_{c 2}}\right)_{0} \frac{\xi_{0}}{l}
$$

(see expression (12)). By the by, the upper critical field $H_{c 2}$ increases also:

$$
\frac{H_{c 2}}{\left(H_{c 2}\right)_{0}}=\frac{\kappa}{\kappa_{0}} \text {. }
$$


Conclusion: the thinner and dirtier the plate is, the larger the interval of a new state's existence is.

Thus, in the interval of fields (21) (see also (22), (23)) there is a liquid state (incompressible liquid, as such a state is called in the theory of fractional quantum Hall effect). This state is explicitly different from Abrikosov vortex lattice. Even if the lattice melted, there still is a transition into a new state because the dependence of energy from the value $\left(1-H / H_{c 2}\right)$ is linear, whereas this dependence remains quadratic, as in (20), for the melted state of Abrikosov lattice, although with other coefficients (the same on value order).

Both transitions on the edges of the interval are transitions of the fist kind with magnetization hops and magnetization is constant inside the interval.

Some things about high-temperature superconductors. As they are a set of weakly connected 2D layers, one can expect that the state discussed here is also possible for them. If it is so, then the corresponding magnetic field interval is to be significantly larger (temperature of transition $T_{c}$ is higher, thickness of layer $d$ is smaller).

My thanks to A.V. Chaplik and M.V. Entin for discussion.

\section{References}

[1] E.G. Batyev, JETP Letters, 11, 554 (1970).

[2] E.G. Batyev, A.Z. Patashinskii, V.L. Pokrovskii, JETP, 46, 2093 (1964).

[3] A.A. Abrikosov, L.P. Gor'kov, I.E. Dzyaloshinskii, Quantum field theoretical methods in statistical physics (2ed., Pergamon, 1965).

[4] G.Blatter, M.V.Feigel'man, V.B.Geshkenbein, A.I.Larkin, V.M.Vinokur, Rev.Mod.Phys., 66, 1125 (1994).

[5] R.B.Laughlin, Phys.Rev.Lett., 50, 1395 (1983). 